This is the author's final, peer-reviewed manuscript as accepted for publication. The publisher-formatted version may be available through the publisher's web site or your institution's library.

\title{
Comparison of antimicrobial susceptibility among Clostridium difficile isolated from an integrated human and swine population in Texas
}

Keri N. Norman, H. Morgan Scott, Roger B. Harvey, Bo Norby, Michael E. Hume

\section{How to cite this manuscript}

If you make reference to this version of the manuscript, use the following information:

Norman, K. N., Scott, H. M., Harvey, R. B., Norby, B., \& Hume, M. E. (2014).

Comparison of antimicrobial susceptibility among Clostridium difficile isolated from an integrated human and swine population in Texas. Retrieved from http://krex.ksu.edu

\section{Published Version Information}

Citation: Norman, K. N., Scott, H. M., Harvey, R. B., Norby, B., \& Hume, M. E. (2014). Comparison of antimicrobial susceptibility among Clostridium difficile isolated from an integrated human and swine population in Texas. Foodborne Pathogens and Disease, 11(4), 257-264.

Copyright: ( Mary Ann Liebert, Inc.

Digital Object Identifier (DOI): doi:10.1089/fpd.2013.1648

Publisher's Link: http://online.liebertpub.com/doi/full/10.1089/fpd.2013.1648

This item was retrieved from the K-State Research Exchange (K-REx), the institutional repository of Kansas State University. K-REx is available at http://krex.ksu.edu 
Comparison of antimicrobial susceptibility among Clostridium difficile isolated from an integrated human and swine population in Texas

\author{
Keri N. Norman ${ }^{1{ }^{*}}$, H. Morgan Scott ${ }^{2}$, Roger B. Harvey ${ }^{3}$, Bo Norby ${ }^{1 \neq}$, \\ Michael E. Hume ${ }^{3}$
}

${ }^{1}$ Department of Veterinary Integrative Biosciences, College of Veterinary Medicine and Biomedical Sciences, Texas A\&M University, College Station, TX 77843, USA

${ }^{2}$ Department of Diagnostic Medicine/Pathobiology, College of Veterinary Medicine, Kansas State University, Manhattan, Kansas 66506, USA

${ }^{3}$ Food and Feed Safety Research Unit, Agricultural Research Service, USDA, College Station, TX 77845, USA

$\dagger$ (current) Department of Biology and Chemistry, Texas A\&M International University, Laredo, TX, 78041, USA

‡ (current) Department of Large Animal Clinical Sciences, College of Veterinary Medicine, Michigan State University, East Lansing, MI 48824, USA

*Corresponding author: Keri N. Norman

Phone: (956) 326-2584; fax: (956) 326-3125; e-mail: keri.norman@tamiu.edu

Running Title: C. difficile antimicrobial susceptibility 


\begin{abstract}
Clostridium difficile can be a major problem in hospitals because the bacterium primarily affects individuals with an altered intestinal flora; this largely occurs through prolonged antibiotic use. Proposed sources of increased community-acquired infections are food animals and retail meats. The objective of this study was to compare the antimicrobial resistance patterns of $C$. difficile isolated from a closed, integrated population of humans and swine to increase understanding of the bacterium in these populations. Swine fecal samples were collected from a vertically flowing swine population consisting of farrowing, nursery, breeding, and grower/finisher production groups. Human wastewater samples were collected from swine worker and non-worker occupational group cohorts. Antimicrobial susceptibility testing was performed on 523 C. difficile strains from the population using commercially available agar diffusion Epsilometer test (Etest $\left.{ }^{\circledR}\right)$ for 11 different antimicrobials. All of the swine and human strains were susceptible to amoxicillin/clavulanic acid, piperacillin/tazobactam, and vancomycin. In addition, all the human strains were susceptible to chloramphenicol. The majority of the human and swine strains were resistant to cefoxitin and ciprofloxacin. Statistically significant differences in antimicrobial susceptibility were found among the swine production groups for ciprofloxacin, tetracycline, amoxicillin/clavulanic acid, and clindamycin. No significant differences in antimicrobial susceptibility were found across human occupational group cohorts. We found that $8.3 \%$ of the swine strains and $13.3 \%$ of the human strains exhibited resistance to metronidazole. The finding of differences in susceptibility patterns between human and swine strains of $C$. difficile, provides evidence that transmission between host species in this integrated population is unlikely.
\end{abstract}




\section{Introduction}

Clostridium difficile is one of the most common causes of nosocomial infections (Vonberg et al., 2008). This anaerobic, Gram-positive bacterium produces spores that are both heat- and alcohol-resistant, and therefore easily transferred between patients and equipment in hospitals and nursing homes (Weir and Flegel, 2005). Clostridium difficile primarily affects individuals with an altered intestinal flora, following periods of prolonged antibiotic use. Patients infected with $C$. difficile experience symptoms ranging from diarrhea to severe cases of colitis that may result in death (Kuijper et al., 2006).

The most common risk factor for $C$. difficile infection is antibiotic use and the risk of infection is increased if the bacteria are resistant to antimicrobial drugs (Gerding, 2004). One of the primary identifying characteristics of the NAP1/ribotype 027/toxinotype III virulent strain, responsible for recent outbreaks, is resistance to clindamycin (Fenner et al., 2008; Kuijper et al., 2008). Patterns of $C$. difficile infection occurring after antibiotic use have been well documented throughout the years. In the 1970s, C. difficile was first associated with the use of clindamycin, leading health care practitioners to decrease use (Gerding, 2004). Fluoroquinolone use began in the late 1980s and in 2001 a study linked ciprofloxacin, a commonly prescribed fluoroquinolone, to C. difficile infection (Yip et al., 2001).

Treatment of $C$. difficile is difficult because of its direct causal relationship with antibiotic use. The two most commonly prescribed antibiotics for C. difficile infection are metronidazole and vancomycin. Resistance of $C$. difficile to metronidazole or vancomycin may lead to treatment failure or relapses of infection (Pituch et al., 2005). Resistance to metronidazole appears to be increasing. Often an initial treatment with 
metronidazole is unsuccessful and subsequent treatment with vancomycin is needed (Huang et al., 2009b). However, the majority of published studies have found no resistance to either vancomycin or metronidazole among $C$. difficile isolated from clinical cases or the environment (Huang et al., 2009a; Taori et al., 2010).

Recent $C$. difficile studies have focused on the apparent increase in outbreaks due to NAP1/ribotype 027/toxinotype III strain infections (Drudy et al., 2008; McDonald et al., 2005) and an increased incidence of community-acquired infections (CDC, 2008; Huang et al., 2009c). By definition, these community-acquired cases have no history of recent hospital exposure (Huang et al., 2009c). Understanding the evolution and source of these strains is crucial for both treatment and prevention. One working hypothesis is that $C$. difficile may be a foodborne pathogen and this hypothesis has been supported by the isolation of $C$. difficile from both food animals and retail meat (Jhung et al., 2008; Keel et al., 2007; Pirs et al., 2008; Rodriguez-Palacios et al., 2007; Simango and Mwakurudza, 2008; Weese et al., 2010; Zidaric et al., 2008). An equally plausible explanation is that $C$. difficile is a ubiquitous organism and humans and food animals are exposed through a common environmental source. Investigating the antimicrobial susceptibility patterns of $C$. difficile from human and food animal populations may help us understand the association between these populations.

The objective of this study was to compare, both within and between host species, the antimicrobial susceptibility patterns of $C$. difficile strains arising from a closed and integrated human and swine population in Texas, U.S.A.

\section{Materials and Methods}


Swine composite fecal samples and human composite wastewater samples were collected from a closed, vertically integrated population in the state of Texas, previously described (Norman et al., 2011; Scott et al., 2005). Details on sample collection and processing and isolation of $C$. difficile have also been previously described (Norman et al., 2009;Norman et al., 2011;Scott et al., 2005).

Antimicrobial sensitivity testing was performed on the 523 C. difficile strains isolated from the population using a commercially available agar diffusion Epsilometer test (Etest ${ }^{\circledR}:$ AB Biodisk ${ }^{\mathrm{TM}}$ North America, Inc., Piscataway, NJ). Eleven different antibiotics were tested; ampicillin, chloramphenicol, tetracycline, amoxicillin/clavulanic acid, imipenem, cefoxitin, metronidazole, ciprofloxacin, clindamycin, piperacillin/tazobactam, and vancomycin.

C. difficile strains were grown anaerobically (chamber conditions: 5\% hydrogen, 5\% carbon dioxide, 90\% nitrogen) for 48 hours on anaerobic Brucella plates (Anaerobe Systems, Walnut, CA) at $37^{\circ} \mathrm{C}$. Colonies from the cultured plate were added to $1 \mathrm{ml}$ of Viande-Levure (VL) broth (5g Tryptose (Becton, Dickinson, and Company (BD), Sparks, MD), 2.5 g yeast extract (BD), 2.5 g sodium chloride (VWR International, West Chester, PA), 1.2 g beef extract (BD), 0.3 g cysteine - $\mathrm{HCl}$ (Thermo Scientific, Rockford, IL), and $1.25 \mathrm{~g}$ dextrose (BD) in $500 \mathrm{ml}$ of de-ionized water) to make a McFarland standard of 0.5. Anaerobic Brucella agar plates were streaked per the instructions of the manufacturer (AB Biodisk ${ }^{\mathrm{TM}}$ North America, Inc.). Two different antibiotic strips were placed on the plate in opposite directions and spaced about $2 \mathrm{~cm}$ apart. Plates were incubated anaerobically at $37^{\circ} \mathrm{C}$ and read at 48 hours, per the manufacturer's instructions (AB Biodisk ${ }^{\mathrm{TM}}$ North America, Inc.). Results were interpreted according to agar dilution 
anaerobic breakpoints provided by the Clinical and Laboratory Standards Institute (CLSI) (Table 1) (CLSI, 2007). Clinical and Laboratory Standards Institute anaerobic breakpoints are not provided for vancomycin or ciprofloxacin. Vancomycin results were interpreted using the CLSI breakpoints for Gram-positive aerobes and ciprofloxacin results were interpreted based on trovafloxacin CLSI breakpoints for Gram-positive anaerobes (Table 1). Quality control strains Bacterioides fragilis (ATCC \#25285) and Bacterioides thetaiotaomicron (ATCC \#29741) were tested and interpreted using the recommended CLSI breakpoints.

Antimicrobial susceptibility profiles for the host species were explored using Fisher's exact test and non-parametric survival analysis. Susceptibility to each antibiotic was coded as binary and explored both between and within host species using Fisher's exact test $(\mathrm{p}<0.05)$ (Stata SE Release 10.1). Isolates with susceptible and intermediate breakpoints were collapsed into one category and resistant isolates were placed into a second category to create binary variables. Non-parametric survival analysis was used to assess within and between host differences in the distribution of minimum inhibitory concentration (MIC) values (Stata SE Release 10.1) (Stegeman et al., 2006). Log-rank tests were used to test the equality of MIC distributions and Kaplan-Meier survival curves were produced to visually compare the distributions (State SE Release 10.1). The MIC values were log transformed in order to improve the graphical interpretation of the data in the Kaplan-Meier survival curves. The lowest MIC concentrations that completely inhibited $50 \%\left(\mathrm{MIC}_{50}\right)$ and $90 \%\left(\mathrm{MIC}_{90}\right)$ of the strains were calculated for each of the antimicrobials. Results 
A total of 523 C. difficile strains were isolated from 5,228 swine fecal and human wastewater samples (Norman et al., 2011). There were 252 strains isolated from 2,936 swine fecal samples, including 175 from the farrowing barn, 14 from the nursery, 26 from breeding swine, and 37 from grower/finisher swine. There were 271 strains isolated from 2,292 human wastewater samples, including 131 from swine workers and 140 from non-workers.

Swine binary

All 252 swine strains were susceptible to amoxicillin/clavulanic acid, piperacillin/tazobactam, and vancomycin (i.e., $100 \%$ susceptible, $0 \%$ resistant). The majority of swine strains were resistant to cefoxitin (95.6\%), ciprofloxacin (86.5\%), and imipenem (58.7\%) (Table 2). Interestingly, 8.3\% were resistant to metronidazole. In unconditional associations, there was a significant difference in susceptibility levels between swine production groups for ciprofloxacin $(\mathrm{p}<0.001)$ and tetracycline $(\mathrm{p}<0.01)$. Strains from the breeding group had the highest prevalence of resistance to tetracycline and the lowest prevalence of resistance to ciprofloxacin.

\section{Human binary}

All 271 human strains were susceptible to amoxicillin/clavulanic acid, piperacillin/tazobactam, chloramphenicol, and vancomycin (i.e., 100\% susceptible, 0\% resistant). The majority of human strains were resistant to cefoxitin (96.3\%) and ciprofloxacin (98.5\%). Also, 13.3\% were resistant to metronidazole. There was no significant difference in susceptibility levels between the occupational group cohorts for any of the antimicrobials.

Comparison of binary between and across host species 
In unconditional analyses when comparing susceptibility between host species, there was significantly $(\mathrm{p}<0.05)$ more resistance to ampicillin, clindamycin, and imipenem among swine strains and there was significantly $(\mathrm{p}<0.05)$ more resistance to ciprofloxacin among human strains (Table 2). There was a significant difference in susceptibility levels across all 523 strains for chloramphenicol $(\mathrm{p}<0.05)$, ciprofloxacin $(\mathrm{p}<0.001)$, clindamycin $(\mathrm{p}<0.001)$, imipenem $(\mathrm{p}<0.001)$, and tetracycline $(\mathrm{p}<0.001)$. Strains from the older swine production groups (grower/finisher and breeding) had a higher prevalence of resistance to chloramphenicol compared to the younger swine production groups (farrowing and nursery) and human occupational group cohorts. Interestingly, the swine breeding production group had the highest prevalence of resistance to tetracycline. There was a significant difference in susceptibility levels among seasons for clindamycin $(\mathrm{p}<0.05)$, imipenem $(\mathrm{p}<0.001)$, and tetracycline $(\mathrm{p}<0.001)$ across all 523 strains. The highest percentage of resistance for clindamycin and imipenem was found in the winter and the highest for tetracycline was found in the spring.

Comparison of MIC values within and between host species

Non-parametric survival analysis was used to assess differences in the distribution of MIC values both within and between host species. Significant $(\mathrm{p}<0.05)$ differences were found among host species with trends towards higher MIC values for imipenem, ampicillin, and clindamycin (Figure 1) in swine strains compared to human strains. Significant $\left(\mathrm{p}^{<0.05}\right)$ trends towards higher MIC values for cefoxitin, ciprofloxacin (Figure 2), amoxicillin/clavulanic acid, piperacillin/tazobactam, metronidazole, and tetracycline were found in human strains compared to swine strains. Key MIC values 
were expressed graphically as $\mathrm{MIC}_{50}$ and $\mathrm{MIC}_{90}$, respectively. A significant difference was found among swine production groups for MIC values for amoxicillin/clavulanic acid $(\mathrm{p}=0.04)$ and clindamycin $(\mathrm{p}=0.04)$ (Figure 3$)$. No significant differences $(\mathrm{p}>0.05)$ were found in susceptibility patterns between human occupational group cohorts.

\section{Discussion}

Decreased antimicrobial susceptibility is an important issue when treating cases of C. difficile in both humans and swine. The association between C. difficile in humans and swine is still unknown and there is evidence both supporting and refuting the possibility of transmission between species. The unique nature of the current study allowed us to explore the antimicrobial susceptibility patterns among C. difficile isolated from an integrated population of humans and swine with little movement in or out of the system. Previous studies have compared antimicrobial susceptibility patterns in human and swine populations; however, the populations were separate and unrelated (Debast et al., 2009; Goorhuis et al., 2008).

When comparing across host species, we found significantly more resistance to ampicillin, clindamycin, and imipenem among swine strains. We also found significantly more resistance to ciprofloxacin among human strains compared to swine strains. A study conducted in the Netherlands comparing swine C. difficile strains found similar antimicrobial susceptibility patterns for clindamycin, ciprofloxacin, metronidazole, and vancomycin with those reported for humans (Debast et al., 2009). We also found similar patterns for metronidazole and vancomycin between human and swine. Another study conducted in the Netherlands also found no difference in antimicrobial susceptibility 
patterns for clindamycin or ciprofloxacin between swine and humans (Goorhuis et al., 2008). The type of strains we recovered may affect the antimicrobial susceptibility profiles and provide a reason for the differences in susceptibility patterns between our study and the two Dutch studies.

In addition to differences found between host species, we also found differences in susceptibility patterns between swine production groups for ciprofloxacin, tetracycline, amoxicillin/clavulanic acid, and clindamycin. We did not assess the antimicrobial usage data among these populations in the current study; however, varying rates of historical usage may be responsible for the significant differences found between host species and among swine production groups.. Alali et al. (2009) found that the relative odds of tetracycline resistance for Escherichia coli were significantly increased for chlortetracycline use in medicated feed across swine production groups in this study population (Alali et al., 2009). Interestingly, the breeding group showed the highest prevalence of resistance to tetracycline and historically varying rates of chlortetracycline or oxytetracycline antibiotic treatment could explain these differences. A recent study on the use of in-feed antimicrobials in swine production found that chlortetracycline had the highest estimated use in both the nursery and grower/finisher production groups (Apley et al., 2012). The few swine that entered our swine population would have been breeding boars and these introduced boars may be responsible for the observed resistance to tetracycline.

The significant differences in susceptibility patterns for clindamycin, imipenem, and tetracycline across seasons in the population (both human and swine) may also be explained by varying rates of antimicrobial use. Reduced susceptibility was observed in 
the winter months for clindamycin and imipenem and in the summer months for tetracycline. This reduction may be reflective of an increase in certain conditions or illnesses that required antibiotic treatments during these seasons. . However, it should be noted that the reduced susceptibility to imipenem observed in the winter months could not be explained by differences in antimicrobial use in the swine population because carbapenems including imipenem are restricted from use in food animals.

Valuable data may be lost when collapsing antimicrobial data into binary categories. In addition to analyzing the binary data, survival analysis was used to assess the data across MIC values. Similar results to the binary analysis were found when comparing across host species. Additionally, significantly increased MIC values were found in human strains to cefoxitin, amoxicillin/clavulanic acid, piperacillin/tazobactam, metronidazole, and tetracycline. Significant MIC differences were also found among swine production groups for amoxicillin/clavulanic acid, and clindamycin. As with the binary data analysis, no significant differences were observed across human occupational group cohorts.

Metronidazole and vancomycin are the two most commonly prescribed antimicrobials for treatment of C. difficile infections in humans. The majority of clinical studies have not found resistance to either metronidazole or vancomycin (Huang et al., 2009a; Taori et al., 2010). All 523 swine and human strains in our study were susceptible to vancomycin. However, we found $8.3 \%$ of swine strains and $13.3 \%$ of human strains were resistant to metronidazole (Figure 4). Three studies have previously reported metronidazole resistance but at a lower prevalence (Brazier et al., 2001; Pelaez et al., 2002; Wong et al., 1999). Clinical studies have also started to report decreasing 
effectiveness of metronidazole for treatment of C. difficile infections (Musher et al., 2005; Pepin et al., 2005). One reason for the higher prevalence of metronidazole resistance found in the current study may be the source and type of $C$. difficile strains. One study has reported a greater prevalence of resistance to metronidazole in ribotype 001 strains compared to 027, 106, and several other ribotypes (Baines et al., 2008).

An interesting note about our samples is that many of the metronidazole resistant strains had MIC values that were initially classified as susceptible at 24 hours. However, at 48 hours there was additional hazy growth on the plate, resulting in an MIC value interpreted as resistant. Others have also reported that certain strains appear to be heteroresistant and subpopulations show resistance to metronidazole at a slow rate (Huang et al., 2010). . The bimodal distribution of the metronidazole MIC values observed in our study would support the theory of a heteroresistant population (Figure 4). Huang et al. also found that metronidazole resistance was lost after three passages of freezing and thawing of these isolates. We ran our Etest ${ }^{\circledR}$ strips using pure cultures from plated enrichments. When we thawed a sample of our frozen strains found previously to be metronidazole resistant, we also found that the strains were now susceptible.

There are several limitations with this study. The study design and sample collection methods do not allow us to form definitive conclusions concerning the transmission of $C$. difficile between humans and swine. The limitations of a crosssectional study are that prevalence is the only measurable outcome and it is difficult to determine if the exposure of interest occurred before the outcome was measured. The human wastewater and swine fecal samples were aggregate samples and not collected on an individual level and results must be presented on the population level rather than the 
individual level. A sample positive for $C$. difficile may be the result of one or many infected individuals. Finally little is known about the dynamics of C. difficile in wastewater samples. Chemicals or compounds present in wastewater may have hindered or promoted the growth, preservation, or detection of C. difficile.

Further research is needed to investigate the possible sources of communityacquired C. difficile infection. The current study provides evidence that occupational and food-borne exposures are less likely sources of community-acquired infections. Genetic sequencing of the strains from these populations would provide further information about the similarity, differences, and evolutionary history between host species. Further research is also needed to investigate the dynamics of metronidazole resistance in $C$. difficile strains.

\section{Conclusion}

The finding of differences in susceptibility patterns between host species and the finding of no difference between human occupational group cohorts provides some evidence that transmission between host species in this integrated population is less likely than within species or from point sources. The antimicrobial usage data for the host populations were not assessed in this study; however, varying rates of usage (especially for swine) may be responsible for differences seen between and within host species. The high level of resistance to metronidazole in both the swine and human wastewater samples is of concern and warrants further investigation.

\section{Funding}


This work was supported by the National Pork Board Grants (06-156 and 08-188) and United States Department of Agriculture Cooperative State Research, Education, and Extension Service National Research Initiative Competitive Grants Program Section 32.1 (Epidemiologic Approaches to Food Safety) (2003-35212-13298).

\section{Transparency declarations}

None to declare

\section{Disclaimer}

"Proprietary or brand names are necessary to report factually on available data; however, the USDA neither guarantees nor warrants the standard of the product, and the use of the name by the USDA implies no approval of the product, and/or exclusion of others that may be suitable.”

\section{References}

Alali WQ, Scott HM, Christian KL, Fajt VR, Harvey RB, and Lawhorn DB. Relationship between level of antibiotic use and resistance among Escherichia coli isolates from integrated multi-site cohorts of humans and swine. Prev Vet Med 2009; $90: 160-7$.

Apley MD, Bush EJ, Morrison RB, Singer RS, and Snelson H. Use estimates of in-feed antimicrobials in swine production in the United States. Foodborne Pathog Dis 2012; 9:272-9. 
Baines SD, O'Connor R, Freeman J, Fawley WN, Harmanus C, Mastrantonio P, Kuijper EJ, and Wilcox MH. Emergence of reduced susceptibility to metronidazole in Clostridium difficile. J Antimicrob Chemother 2008; 62:1046-52.

Brazier JS, Fawley W, Freeman J, and Wilcox MH. Reduced susceptibility of Clostridium difficile to metronidazole. J Antimicrob Chemother 2001; 48:741-2.

[CDC] Centers for Disease Control and Prevention. Surveillance for communityassociated Clostridium difficile--Connecticut, 2006. MMWR Morb Mortal Wkly Rep 2008; 57:340-3.

Debast SB, van Leengoed LA, Goorhuis A, Harmanus C, Kuijper EJ, and Bergwerff AA. Clostridium difficile PCR ribotype 078 toxinotype V found in diarrhoeal pigs identical to isolates from affected humans. Environ Microbiol 2009; 11:505-11.

Drudy D, Goorhuis B, Bakker D, Kyne L, van den Berg R, Fenelon L, Fanning S, and Kuijper EJ. Clindamycin-resistant clone of Clostridium difficile PCR Ribotype 027, Europe. Emerg Infect Dis 2008; 14:1485-7.

Fenner L, Widmer AF, Stranden A, Conzelmann M, Goorhuis A, Harmanus C, Kuijper EJ, and Frei R. First cluster of clindamycin-resistant Clostridium difficile PCR ribotype 027 in Switzerland. Clin Microbiol Infect 2008; 14:514-5.

Gerding DN. Clindamycin, cephalosporins, fluoroquinolones, and Clostridium difficileassociated diarrhea: this is an antimicrobial resistance problem. Clin Infect Dis 2004; 38:646-8.

Goorhuis A, Bakker D, Corver J, Debast SB, Harmanus C, Notermans DW, Bergwerff AA, Dekker FW, and Kuijper EJ. Emergence of Clostridium difficile infection 
due to a new hypervirulent strain, polymerase chain reaction ribotype 078 . Clin Infect Dis 2008; 47:1162-70.

Huang H, Fang H, Weintraub A, and Nord CE. Distinct ribotypes and rates of antimicrobial drug resistance in Clostridium difficile from Shanghai and Stockholm. Clin Microbiol Infect 2009a; 15:1170-3.

Huang H, Weintraub A, Fang H, and Nord CE. Antimicrobial resistance in Clostridium difficile. Int J Antimicrob Agents 2009b; 34:516-22.

Huang H, Weintraub A, Fang H, and Nord CE. Community acquired Clostridium difficile infection due to a moxifloxacin susceptible ribotype 027 strain. Scand J Infect Dis 2009c; 41:158-9.

Huang H, Weintraub A, Fang H, Wu S, Zhang Y, and Nord CE. Antimicrobial susceptibility and heteroresistance in Chinese Clostridium difficile strains. Anaerobe 2010; 16:633-5.

Jhung MA, Thompson AD, Killgore GE, Zukowski WE, Songer G, Warny M, Johnson S, Gerding DN, McDonald LC, and Limbago BM. Toxinotype V Clostridium difficile in humans and food animals. Emerg Infect Dis 2008; 14:1039-45.

Keel K, Brazier JS, Post KW, Weese S, and Songer JG. Prevalence of PCR ribotypes among Clostridium difficile isolates from pigs, calves, and other species. J Clin Microbiol 2007; 45:1963-4.

Kuijper EJ, Barbut F, Brazier JS, Kleinkauf N, Eckmanns T, Lambert ML, Drudy D, Fitzpatrick F, Wiuff C, Brown DJ, Coia JE, Pituch H, Reichert P, Even J, Mossong J, Widmer AF, Olsen KE, Allerberger F, Notermans DW, Delmee M, Coignard B, Wilcox M, Patel B, Frei R, Nagy E, Bouza E, Marin M, Akerlund T, 
Virolainen-Julkunen A, Lyytikainen O, Kotila S, Ingebretsen A, Smyth B, Rooney P, Poxton IR, and Monnet DL. Update of Clostridium difficile infection due to PCR ribotype 027 in Europe, 2008. Euro Surveill 2008; 13.

Kuijper EJ, Coignard B, and Tull P. Emergence of Clostridium difficile-associated disease in North America and Europe. Clin Microbiol Infect 2006; 12 Suppl 6:218.

McDonald LC, Killgore GE, Thompson A, Owens RC, Jr., Kazakova SV, Sambol SP, Johnson S, and Gerding DN. An epidemic, toxin gene-variant strain of Clostridium difficile. N Engl J Med 2005; 353:2433-41.

Musher DM, Aslam S, Logan N, Nallacheru S, Bhaila I, Borchert F, and Hamill RJ. Relatively poor outcome after treatment of Clostridium difficile colitis with metronidazole. Clin Infect Dis 2005; 40:1586-90.

Norman KN, Harvey RB, Scott HM, Hume ME, Andrews K, and Brawley AD. Varied prevalence of Clostridium difficile in an integrated swine operation. Anaerobe 2009; 15:256-60.

Norman KN, Scott HM, Harvey RB, Norby B, Hume ME, and Andrews K. Prevalence and genotypic characteristics of Clostridium difficile in a closed and integrated human and swine population. Appl Environ Microbiol 2011; 77:5755-60.

Pelaez T, Alcala L, Alonso R, Rodriguez-Creixems M, Garcia-Lechuz JM, and Bouza E. Reassessment of Clostridium difficile susceptibility to metronidazole and vancomycin. Antimicrob Agents Chemother 2002; 46:1647-50. 
Pepin J, Alary ME, Valiquette L, Raiche E, Ruel J, Fulop K, Godin D, and Bourassa C. Increasing risk of relapse after treatment of Clostridium difficile colitis in Quebec, Canada. Clin Infect Dis 2005; 40:1591-7.

Pirs T, Ocepek M, and Rupnik M. Isolation of Clostridium difficile from food animals in Slovenia. J Med Microbiol 2008; 57:790-2.

Pituch H, Obuch-Woszczatynski P, Wultanska D, Meisel-Mikolajczyk F, and Luczak M. A survey of metronidazole and vancomycin resistance in strains of Clostridium difficile isolated in Warsaw, Poland. Anaerobe 2005; 11:197-9.

Rodriguez-Palacios A, Staempfli HR, Duffield T, and Weese JS. Clostridium difficile in retail ground meat, Canada. Emerg Infect Dis 2007; 13:485-7.

Scott HM, Campbell LD, Harvey RB, Bischoff KM, Alali WQ, Barling KS, and Anderson RC. Patterns of antimicrobial resistance among commensal Escherichia coli isolated from integrated multi-site housing and worker cohorts of humans and swine. Foodborne Pathog Dis 2005; 2:24-37.

Simango C and Mwakurudza S. Clostridium difficile in broiler chickens sold at market places in Zimbabwe and their antimicrobial susceptibility. Int J Food Microbiol 2008; $124: 268-70$.

Stegeman JA, Vernooij JC, Khalifa OA, Van den Broek J, and Mevius DJ. Establishing the change in antibiotic resistance of Enterococcus faecium strains isolated from Dutch broilers by logistic regression and survival analysis. Prev Vet Med 2006; 74:56-66. 
Taori SK, Hall V, and Poxton IR. Changes in antibiotic susceptibility and ribotypes in Clostridium difficile isolates from southern Scotland, 1979-2004. J Med Microbiol 2010; 59:338-44.

Vonberg RP, Reichardt C, Behnke M, Schwab F, Zindler S, and Gastmeier P. Costs of nosocomial Clostridium difficile-associated diarrhoea. J Hosp Infect 2008; 70:1520.

Weese JS, Reid-Smith RJ, Avery BP, and Rousseau J. Detection and characterization of Clostridium difficile in retail chicken. Lett Appl Microbiol 2010; 50:362-5.

Weir E and Flegel K. Protecting against Clostridium difficile illness. CMAJ 2005; 172:1178.

Wong SS, Woo PC, Luk WK, and Yuen KY. Susceptibility testing of Clostridium difficile against metronidazole and vancomycin by disk diffusion and Etest. Diagn Microbiol Infect Dis 1999; 34:1-6.

Yip C, Loeb M, Salama S, Moss L, and Olde J. Quinolone use as a risk factor for nosocomial Clostridium difficile-associated diarrhea. Infect Control Hosp Epidemiol 2001; 22:572-5.

Zidaric V, Zemljic M, Janezic S, Kocuvan A, and Rupnik M. High diversity of Clostridium difficile genotypes isolated from a single poultry farm producing replacement laying hens. Anaerobe 2008; 14:325-7. 